\title{
The First Case Report of a Rare Gastric Plexiform Fibromyxoma: Histological Images With an Infiltrative Growth Pattern of Diffuse Infiltration in Stomach.
}

\section{Zhenyu Li}

Chongqing University

Qingming Jiang

Chongqing University

Dongfang Guo

Chongqing University

Yangling Peng

Chongqing University

Jing Zhang

Chongqing University

Xinyu Chen ( $\nabla$ chenxinyu9739@126.com )

Chongqing University https://orcid.org/0000-0002-7588-220X

\section{Case Report}

Keywords: Plexiform Fibromyxoma, multinodular, diffuse growth, stomach, SMA, case report

Posted Date: March 31st, 2021

DOI: https://doi.org/10.21203/rs.3.rs-362389/v1

License: (c) (i) This work is licensed under a Creative Commons Attribution 4.0 International License.

Read Full License 


\section{Abstract}

Background: Plexiform Fibromyxoma (PF) of the stomach is a very rare mesenchymal tumor of the gastrointestinal tract. We report the first case of PF which was confirmed after surgical resection pathologically.

Case summary: The tumor was characterised microscopically infiltrative and diffuse growth into the smooth muscle bundles of the muscularis propria by multinodular and plexiform within myxoid stroma. Immunohistochemistry analysis revealed that the tumor cells were positive or weakly positive for smooth muscle actin (SMA), vimentin and H-caldesmon, and negative for desmin, CD117, CD34, CK-20, Pan-CK, Dog1, S100, ER, PR, and CD10. No mutations of C-kit and platelet-derived growth factor receptor alpha (PDGFRA) were detected in our case. No genetic disruption of glioma-associated oncogene homolog 1 (GLI1) was detected by fluorescence in situ hybridization (FISH).

Conclusion: So we mainly relied on the morphological and immunohistochemistry findings and made the final diagnosis of PF.

\section{Introduction}

Plexiform Fibromyxoma is a rare mesenchymal tumor of the gastrointestinal (GI) tract. It was first reported by Takahashi et al. in $2007^{[1]}$. And then it was officially recognized as a distinct entity among benign mesenchymal gastric tumors in the 2010 WHO Classification of Tumors of the Digestive System ${ }^{[2]}$. There were more than 120 cases in the literature so far. It is characterised by a multinodularplexiform involvement of gastric wall of bland spindle cells in myxoid and/or fibromyxoidstroma ${ }^{[3]}$. Here, we present the histologic features of gastric plexiform fibromyxoma with two different growth pattern from surgical resection specimen, and discuss the differential diagnoses of this tumor.

\section{Case Presentation}

A 65 years old male was found to have hepatic cysts during physical examination more than 10 years ago. And the patient presented with epigastric pain and discomfort without obvious inducement half a year ago.

Then, he was admitted and underwent abdominal computed tomography (CT). The CT demonstrated multiple cystic space occupation of the liver and a $2.6 \mathrm{~cm}$ mass with prominent contrast enhancement at gastric body. It was suspected to be a (Gastrointestunal stromal tumors) GIST (Fig. 1A-D).

Esophagogastroduodenoscopy revealed multiple gastric polyps and submucosal eminence of the gastric body (Fig. 1E).

Laboratory tests revealed an increase of platelet large cell ratio (P-LCR, 50.10\%), mean platelet volume (MPV, $13.10 \mathrm{fL}$ ), platelet distribution width (PDW, $19.00 \mathrm{fL})$, uric acid (433.70 $\mu \mathrm{mol} / \mathrm{L}), \beta 2$-microglobulin 
$(3.50 \mathrm{mg} / \mathrm{L})$, cystatin C $(1.4 \mathrm{mg} / \mathrm{L})$ and decrease of total protein $(63.30 \mathrm{~g} / \mathrm{L})$, serum complement C1q (143.90 mg/L). The tumor biomarkers such as neuron-specific enolase (NSE, $9.77 \mathrm{ng} / \mathrm{ml})$, CA199 (37.26 $\mathrm{U} / \mathrm{ml})$ and CA50 (33.51 IU/ml) showed slightly higher.

Histopathological findings of the resected mass revealed a submucosal multinodular tumor measuring $1.9 \times 1.4 \mathrm{~cm}$. The tumor showed microscopically infiltrative growth into the smooth muscle bundles of the muscularispropria, some tumor cells were epithelioid and diffused in growth (Fig. 2A-C). There were loose myxoid and cellular areas of the tumor admixed with smooth muscle cells on high-powered microscopic fields (Fig. 2D). Immunohistochemical staining showed that the tumor cells were positive for smooth muscle actin (SMA), vimentin and H-caldesmon, but negative for Desmin, CD117, CD34, CK-20, Dog1, S100, Pan-CK, ER, PR, and CD10. In addition, CD34 highlighted a rich capillary network but was negative in tumor cells. The Ki-67 labeling index was less than $5 \%$ (Fig. 3). Besides, there was another growth pattern in this case. The tumor was characterised microscopically infiltrative growth into the smooth muscle bundles of the muscularis propria by diffusing on other tissue slice (Fig. 4A-C), no like the infiltrative growth pattern by multinodular and plexiform before, and the myxoid stroma was not that loose. Immunohistochemical staining showed that the tumor cells were positive for SMA, vimentin and $\mathrm{H}$ caldesmon but negative for Desmin, CD117, CD34, CK-20 Dog1, S100, Pan-CK, ER, PR, and CD10, just like the Fig. 2. In addition, CD34 highlighted a rich capillary network but was negative in tumor cells. The ki-67 labeling index was less than $5 \%$ (Fig. 5). In order to verify the real component of the tumor cells in the diffusing pattern, we used some molecular pathology methods. The GLI1 break-apart probe by FISH showed no positive finding in the tumor cells (Fig. 6A). No mutations were found in C-kit (exons 9, 11, 13,17 ) or PDGFRA (exons 12,18) gene (Fig. 6B-C). The diagnostic process of this case in our department of pathology (Fig. 7). Under the HE staining by microscope, the morphology of the tumor cells and growth pattern looked like a GIST, PF or gastroblastoma. And the tumor cells were negative for Pan-CK and CD10 by immunohistochemistryl, we firstly excluded the diagnosis of gastroblastoma. Secondly, the tumor cells were negative for CD117, CD34 and Dog1 by immunohistochemistryl and were unmutated on C-kit or PDGFRA gene, we afterwards excluded the diagnosis of GIST. The last, according to the tumor cells were positive for SMA, vimentin and H-caldesmon and the negative for desmin, besides the GLI-1 disruption (-), we made the final diagnosis for PF. The final diagnosis was made by Dr. Xinyu Chen, Dr. Zhenyu Li, Dr. Qingming Jiang, and Dr. Dongfang Guo of the department of pathology in Chongqing University Cancer Hospital.

The patient remained under careful observation by endoscopy and CT follow-up, and there was no recurrence or metastasis in the 12 months follow-up. The patient got appropriate perspective including the assessment and the episode of care in every 3 months.

\section{Discussion}

Plexiform Fibromyxoma of the stomach is a benign tumor of gastrointestinal tract with more than 120 cases reported in the literatures ${ }^{[4,5,6,7,8]}$. It is mainly found in the gastric antrum, On immunohistochemistry, the tumor cells of PF are positive for SMA and negative for c-kit, CD34, S-100 
protein, epithelial membrane antigen (EMA), and desmin ${ }^{[9]}$. In our case, the morphology of the tumor cells and growth pattern looked like a GIST, PF or gastroblastoma. Interestingly, the tumor cells were under two different growth pattern: infiltrative growth by multinodular and plexiform within myxoid stroma, and diffuse growth without nodosity. We choose two different tissue slices to immunohistochemistry and molecular pathological methods. Because of the negative outcomes of Pan-CK and CD10 for tumor cells by immunohistochemistry, we firstly excluded the diagnosis of gastroblastoma. And then, according to the negative results for CD117, CD34 and Dog1 and C-kit or PDGFRA gene, we afterwards excluded the diagnosis of GIST. The tumor cells were positive for SMA, vimentin and $\mathrm{H}$-caldesmon and the negative for desmin, besides the GLI-1 disruption (-), we made the final diagnosis for PF. Recent research found the gene GLI1 translocation was present in a subgroup of PFs, but not all of the PFs. However, a 5-year-old male was diagnosed PF without GLI1 breaking apart ${ }^{[10,11]}$. And our case did not detect the GLI1 gene breaking up unfortunately. Reportedly, C-kit or PDGFRA mutations were identified in GIST, but not in PF yet ${ }^{[12,13,14]}$. Finnally, the diagnosis of PF mainly relies on the morphological, immunohistochemistry and molecular pathological outcomes.

PF has a benign clinical behavior, and with no reports of either malignant transformation or metastatic spread. According to the current literature, surgical resection or partial gastrectomy is a major treatment for dealing with PF when it occurs in the stomach ${ }^{[15,16]}$. There also have been reports of endoscopic resection for small tumor ${ }^{[17]}$. Due to its rarity, more cases and close follow-up observation are needed.

In summary, we present an unusual and rare patient of PF whose tumor cells have two different growth pattern which is first reported. Although the tumor has distinct histological and immunohistochemical features, awareness is important for its diagnosis. It is worth mentioning that PF tumor cells not only have infiltrative growth pattern by multinodular and plexiform within myxoid stroma, but also can grow diffusely and largely.

\section{Abbreviations}

PF: Plexiform Fibromyxoma; SMA: smooth muscle actin; PDGFRA: growth factor receptor alpha; GLI1: glioma-associated oncogene homolog 1; FISH: fluorescence in situ hybridization; GI: gastrointestinal; CT: computed tomography; GIST: Gastrointestunal stromal tumors; P-LCR: platelet large cell ratio; MPV: mean platelet volume; PDW: platelet distribution width; NSE: neuron-specific enolase; EMA: epithelial membrane antigen; CK: cytokeratin; ER: estrogen receptor; PR: progesterone receptor; WHO: World Health Organization.

\section{Declarations}

\section{Ethics approval and consent to participate}

Informed consent was obtained in this case, and protocols were approved by the Ethics approval of Chongqing University Cancer Hospital. The patient provided informed consent for the publication of this 
report and any accompanying images.

\section{Availability of data and materials}

The datasets used or analysed during the current study are available from the corresponding author on reasonable request.

\section{Competing interests}

The authors declare that there is no conflict of interest.

\section{Funding}

No funding information.

\section{Author's contributions}

ZL contributed to acquisition, analysis and interpretation of patient data and the drafting of the manuscript. DG and YP contributed to the acquire the CT and esophagogastroduodenoscopy data. JZ contributed to the immunohistochemistry and molecular pathological methods. QJ and XC gave the final approval of the report. All authors read and approved the final manuscript.

\section{Acknowledgements}

Not applicable.

\section{References}

1. Takahashi Y, Shimizu S, Ishida T, et al. Plexiform angiomyxoid myofibroblastic tumor of the stomach. Am J Surg Pathol. 2007, 31(5): 724-728.

2. Lien Spans, Christopher Dm Fletcher, Cristina R Antonescu, et al. Recurrent MALAT1-GLI1 oncogenic fusion and GLI1 up-regulation define a subset of plexiform fibromyxoma. J Pathol. 2016, 239(3): 335-43.

3. Jiang Tang, Feng Liu. Plexiform Fibromyxoma: A Rare Mesenchymal Tumor Found in the Esophagus. Am J Gastroenterol. 2020, 115(5): 648.

4. Hsuan-An Su, Hsu-Heng Yen, Chih-Jung Chen, et al. An Update on Clinicopathological and Molecular Features of Plexiform Fibromyxoma. Can J Gastroenterol Hepatol. 2019, 2019: 3960920.

5. Kinga Szurian, Holger Till, Eva Amerstorfer, et al. Rarity among benign gastric tumors: Plexiform fibromyxoma - Report of two cases. World J Gastroenterol. 2017, 23(31): 5817-5822.

6. JinpingLai, Jesse L.Kresak, DengfengCao, et al. Gastric Plexiform Fibromyxoma: A Great Mimic of Gastrointestinal Stromal Tumor (GIST) and Diagnostic Pitfalls. J Surg Res. 2019, 239: 76-82. 
7. Mustafa Erdem Arslan, Hua Li, Timothy A Jennings, et al. Frequency of Plexiform Fibromyxoma relative to gastrointestinal stromal tumor: A single center study. Ann Diagn Pathol. 2020, 48: 151568.

8. Yu-Pu Hong, Jia Yu, Chen-Yang Wang, et al. Plexiform Fibromyxoma of the Stomach. $J$ Gastrointest Surg. 2020, 24(4): 909-912.

9. Kang Y, Jung W, Do I G, et al. Plexiform angiomyxoid myofibroblastic tumor of the stomach: report of two cases and review of the literature. Korean J Pathol. 2012, 46(3): 292-296.

10. Spans L, Fletcher C D, Antonescu C R, et al. Recurrent MALAT1-GLI1 oncogenic fusion and GLI1 upregulation define a subset of plexiform fibromyxoma. J Pathol. 2016, 239(3): 335-343.

11. Li J, Gao H, Lv M, et al. Gastric plexiform fibromyxoma: A rare case in a 5-year-old male. Pediatr Blood Cancer. 2019, 66(5): e27638.

12. Gerardo A Vitiello, Timothy G Bowler, Mengyuan Liu, et al. Differential immune profiles distinguish the mutational subtypes of gastrointestinal stromal tumor. J Clin Invest. 2019, 129(5): 1863-1877.

13. Guiming Hu, Huiping Chen, Qiuyu Liu, et al. Plexiform fibromyxoma of the stomach: a clinicopathological study of 10 cases. Int J Clin Exp Pathol. 2017, 10(11): 10926-10933.

14. Aeri Kim, Young Kyung Bae, Hyeong Chan Shin, et al. Plexiform angiomyxoid myofibroblastic tumor of the stomach: a case report. J Korean Med Sci. 2011, 26(11): 1508-11.

15. Markku Miettinen, Hala R Makhlouf, Leslie H Sobin, et al. Plexiform fibromyxoma: a distinctive benign gastric antral neoplasm not to be confused with a myxoid GIST. Am J Surg Pathol. 2009, 33(11): 1624-32.

16. Biyan Lu, Weibiao Ye, Huanliang Liu, et al. A Rare Gastric Tumor in a Young Woman. Gastric Plexiform Angiomyxoid Myofibroblastic Tumor. Gastroenterology. 2015, 149(2): 294-5.

17. Fukazawa $\mathrm{M}, \mathrm{Koga} \mathrm{H}$, Hiroshige $\mathrm{S}$, et al. Pediatric plexiform fibromyxoma: A PRISMA-compliant systematic literature review. Medicine (Baltimore). 2019, 98(3): e14186.

\section{Figures}



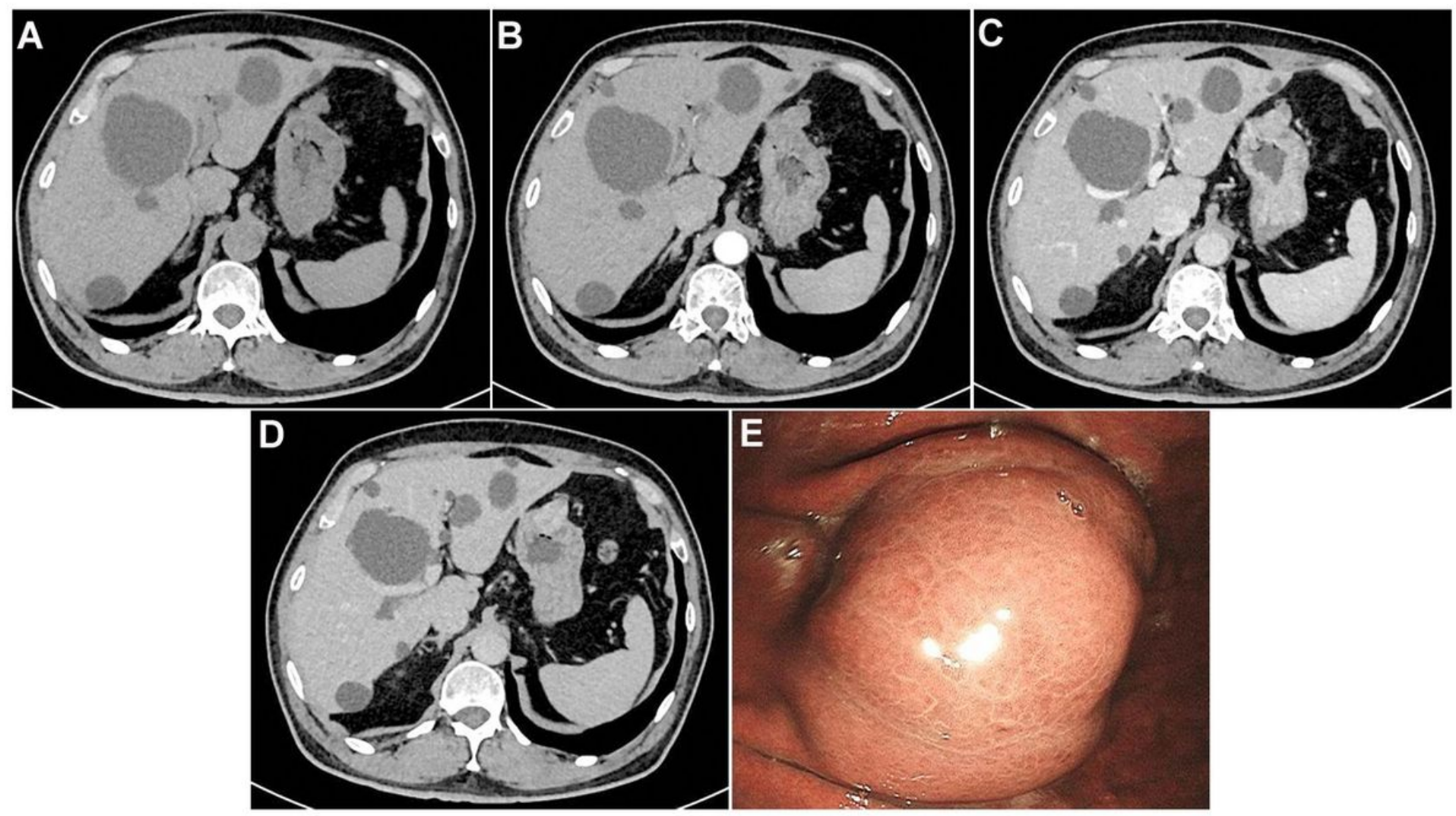

Figure 1

(A) The lesion exhibited with uniform density in axial unenhanced CT image, and with continuous inhomogeneous enhancement in (B) artery phase, (C) venous phase, and (D) delayed phase. (E) Esophagogastroduodenoscopy revealed multiple gastric polyps and submucosal eminence of the gastric body. 


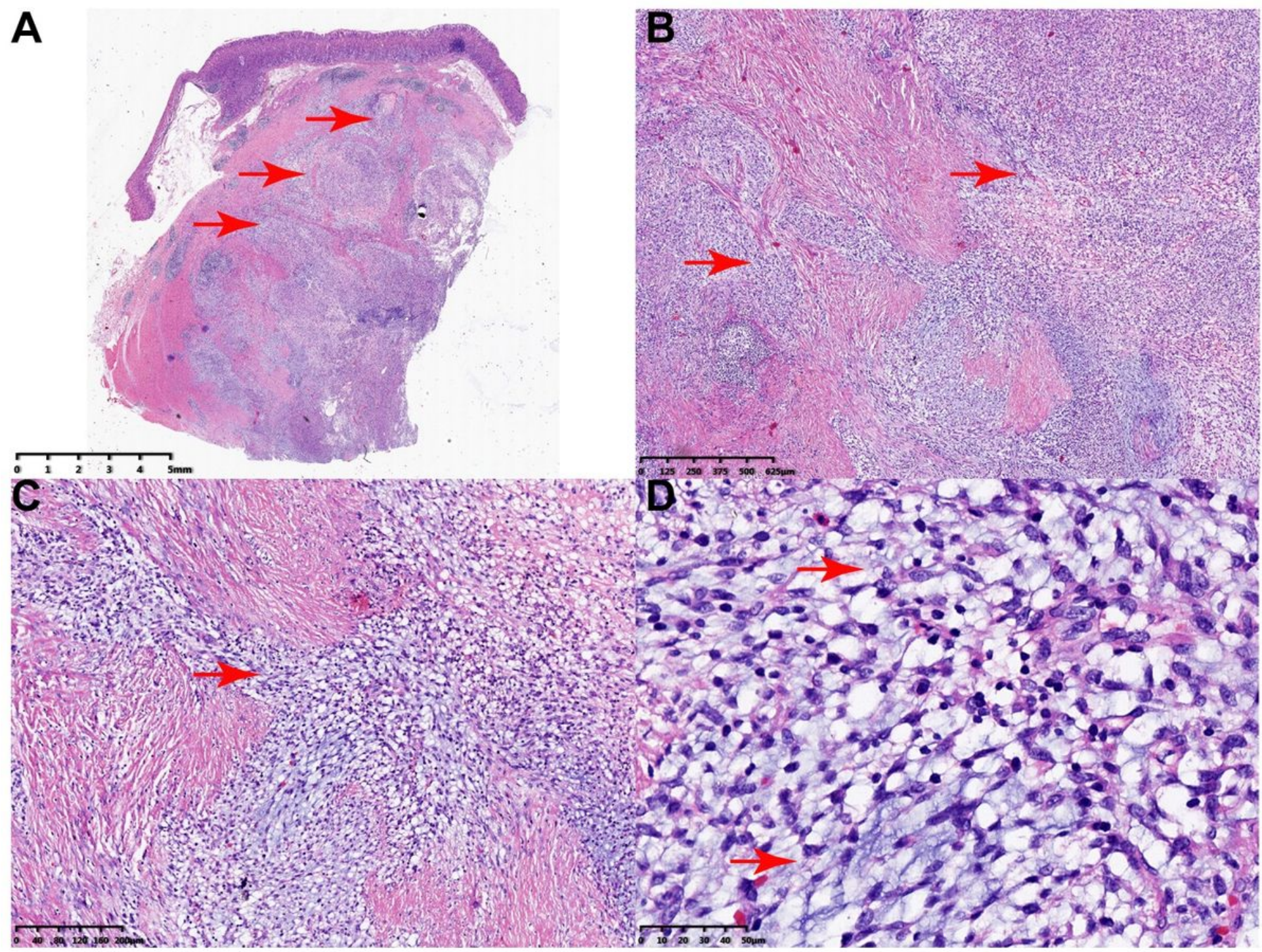

Figure 2

(A) The tumor was characterised microscopically infiltrative growth into the smooth muscle bundles of the muscularis propria by multinodular and plexiform $(\times 1)$. (B-C) Infiltrative growth pattern into the smooth muscle bundles of the muscularispropria, some tumor cells were epithelioid and diffused in growth $(\times 100)$ and $(\times 200)$. (D) Abundant mucus around the proliferative spindle cells $(\times 400)$. (The red arrow indicated the multinodular and plexiform background, the infiltrative growth pattern and the mucus). 


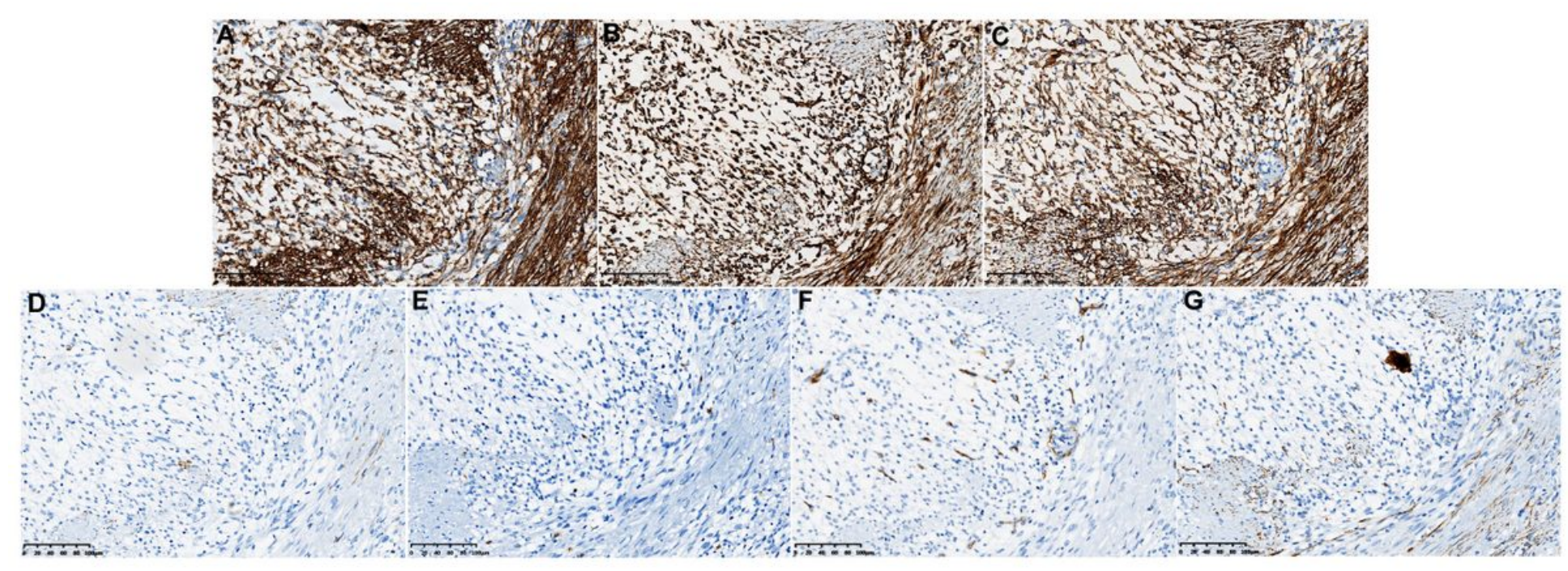

\section{Figure 3}

Immunohistochemical staining shown the tumor cells were positive for a-SMA (A), vimentin (B) and $\mathrm{H}$ caldesmon (C), and negative for Desmin (D), CD117 (E), CD34 (F) and CK-20 (G).
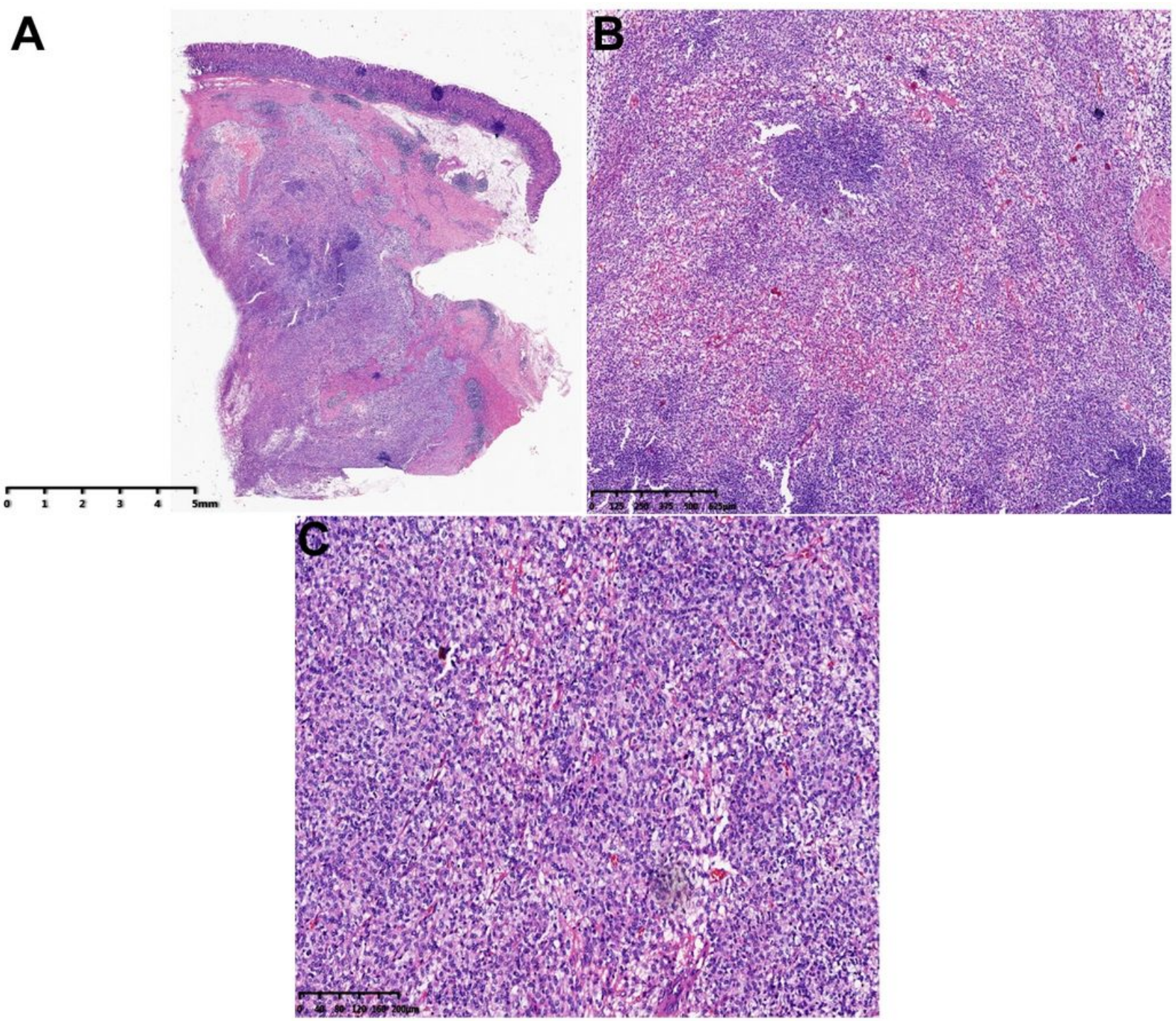


\section{Figure 4}

(A) The tumor was characterised microscopically infiltrative growth into the smooth muscle bundles of the muscularis propria by diffusing $(\times 1)$. (B-C) Infiltrative growth pattern into the smooth muscle bundles of the muscularispropria $(\times 100)$ and $(\times 200)$.

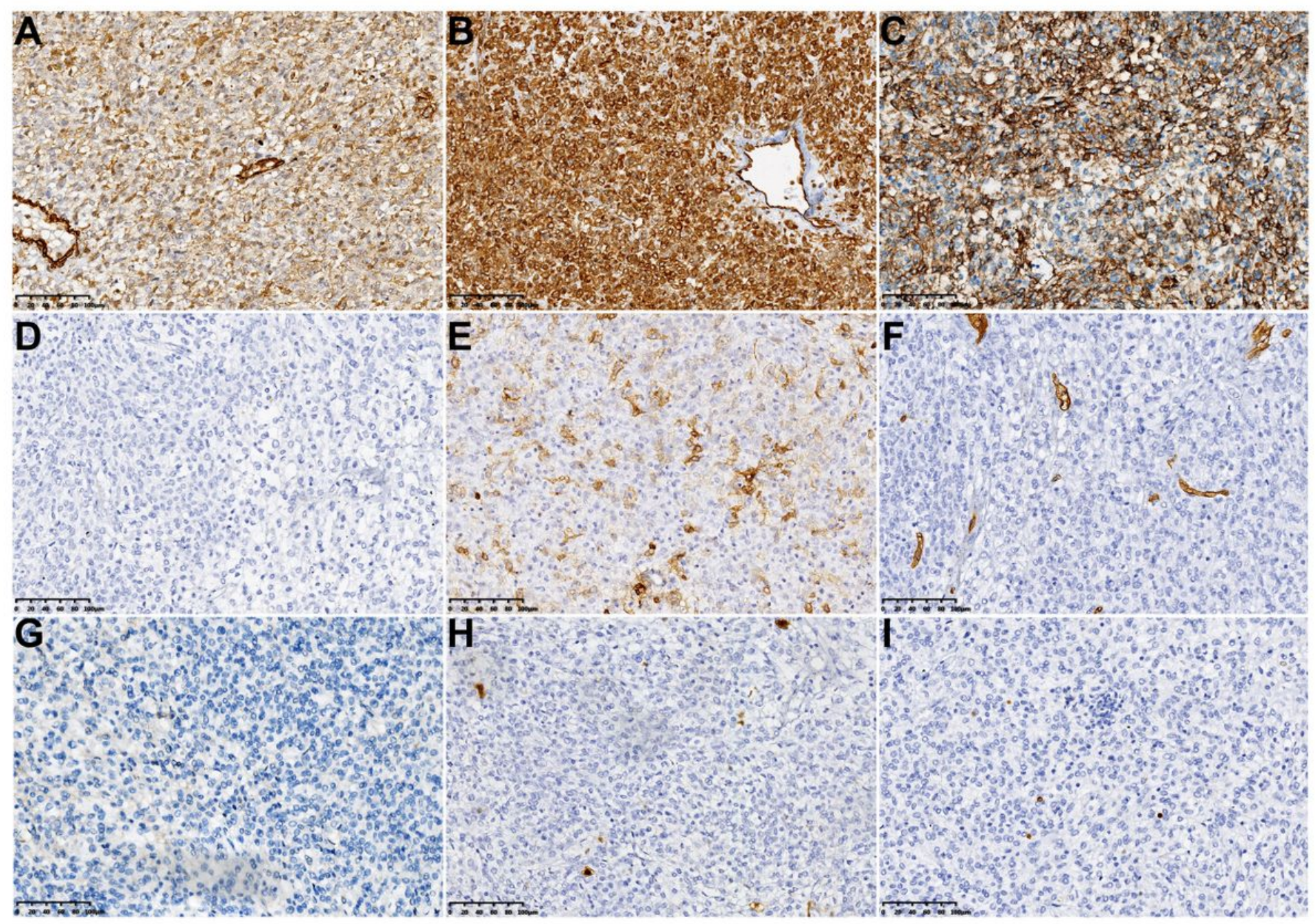

\section{Figure 5}

Immunohistochemical staining shown the tumor cells were positive for a-SMA (A), vimentin (B) and $\mathrm{H}$ caldesmon (C), and negative for Desmin (D), CD117(E), CD34 (F) and CK-20 (G), S100 (H) and ki-67 (I). 


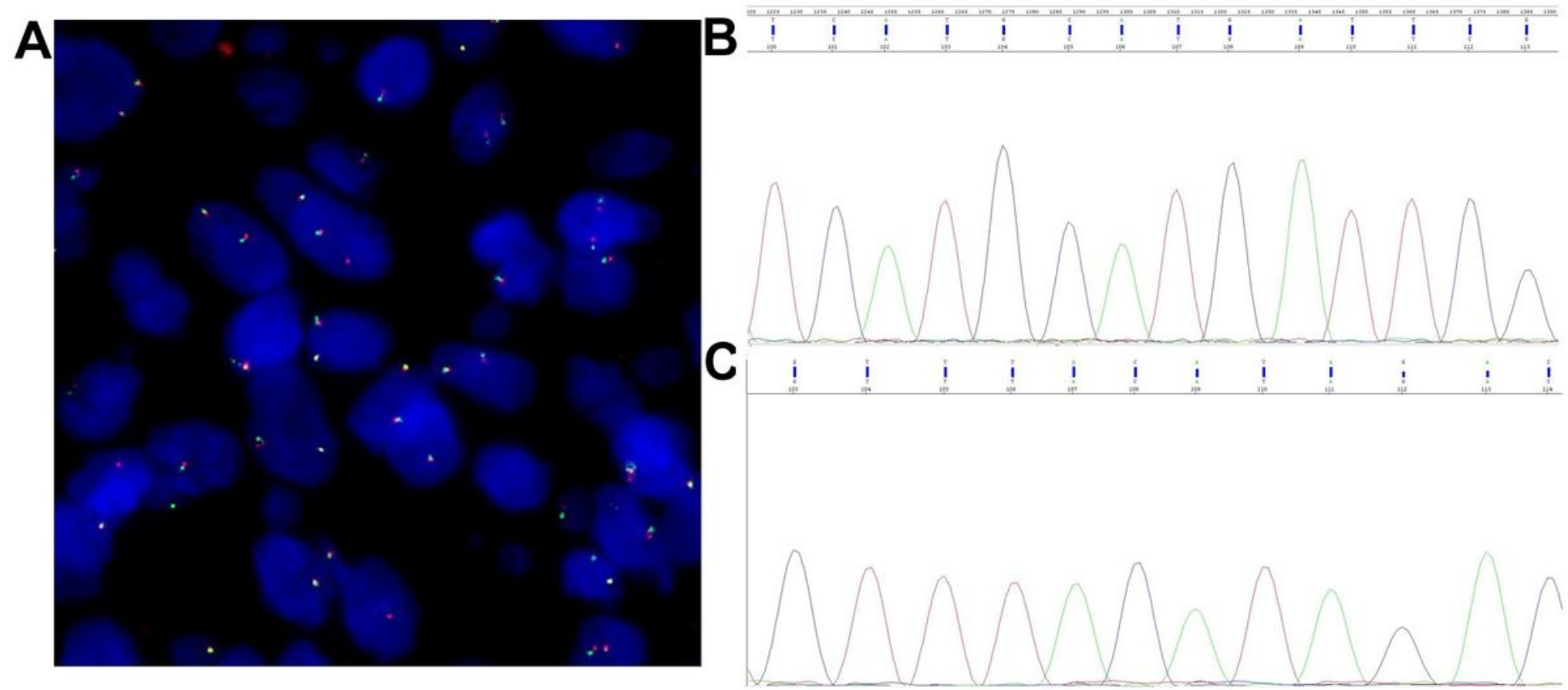

Figure 6

(A) No positive finding of the GLI1 break-apart probe in the tumor cells by FISH. (B-C) The tumor cells were not mutated in C-kit (exons $9,11,13,17$ ) or PDGFRA (exons 12, 18).
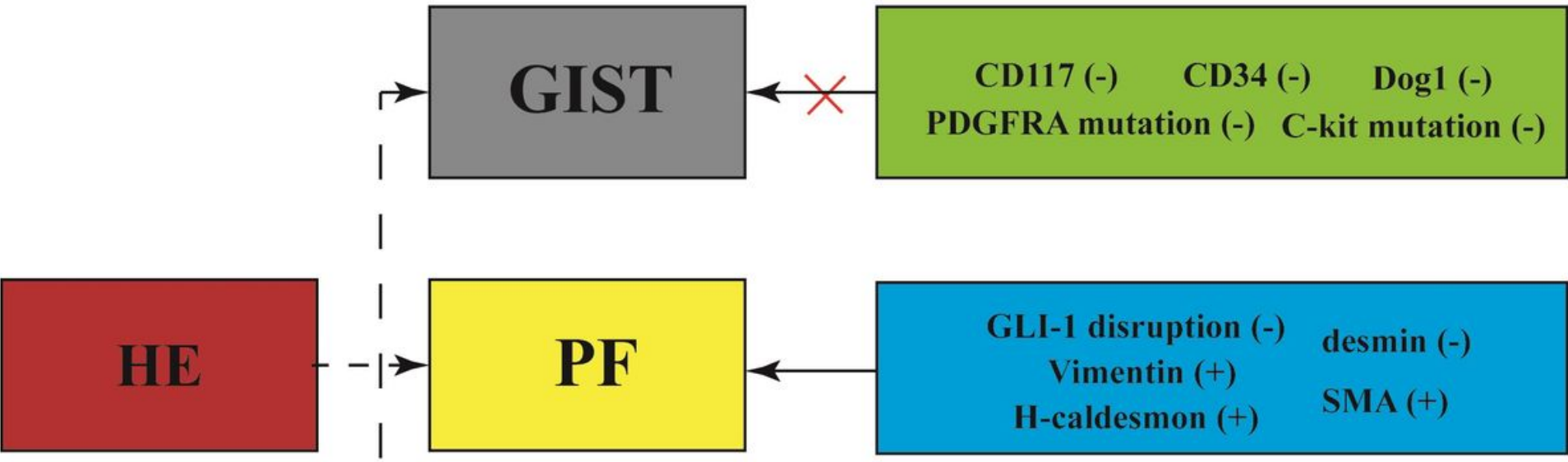

\section{GLI-1 disruption (-) desmin (-) Vimentin $(+)$ H-caldesmon $(+)$ $\operatorname{SMA}(+)$}

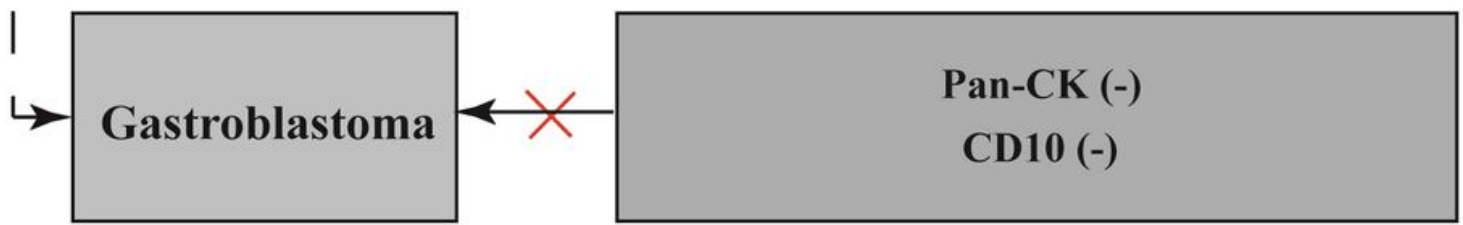

Figure 7

The diagnostic process of this case in our department of pathology.

\section{Supplementary Files}


This is a list of supplementary files associated with this preprint. Click to download.

- ChenCAREchecklistEnglish2013.pdf 